\title{
Effect of Different Ventricular Arrhythmia Origin on Cardiac Sounds Variability Using M-mode Signals Representation
}

\author{
Raúl Ortiz-Puente ${ }^{1}$, Margarita Sanromán-Junquera ${ }^{1}$, Sergio Muñoz-Romero ${ }^{1,2}$, Mercedes Ortiz $^{4}$, \\ Mariano Barbero-Puchades ${ }^{3}$, Rebeca Goya-Esteban ${ }^{1}$, José Luis Rojo-Álvarez ${ }^{1,2}$, \\ Jesús Almendral-Garrote ${ }^{4}$ \\ ${ }^{1}$ Department of Signal Theory and Communications, Telematics and Computing, \\ Universidad Rey Juan Carlos, Madrid, Spain \\ ${ }^{2}$ Center for Computational Simulation, Universidad Politécnica de Madrid, Spain \\ ${ }^{3}$ LivaNova, Barcelona, Spain \\ ${ }^{4}$ Hospital Universitario HM Montepríncipe, Boadilla del Monte, Madrid, Spain
}

\begin{abstract}
In recent years, interest has been paid to the identification of the arrhythmia anatomical origin in ventricular tachycardia from available information in the patient, including ECG morphology and electrograms stored in implantable devices. In this work, we scrutinize the differences in morphology from cardiac sounds. For this purpose, we compared the spatial and temporal similarity in cardiac sounds recorded during electrical intracardiac stimulation, in terms of the so-called M-mode representation for cardiac signals. A protocol was set in $5 \mathrm{pa}$ tients referred to electrophysiological study. Cardiac pacing was made at $400 \mathrm{~ms}$ from different points in the left ventricle. During stimulation, we recorded: (1) the ECG signals from 12 leads; (2) the spatial coordinates for the pacing site, by using an intracardiac navigation system; (3) the heart sounds, by using a high-fidelity recording system. After visual-supervised correction, M-mode maps represented an aligned and clearly represented the variations due to distance and substrate in a consistent view. The same signal processing was followed for the cardiac sounds, showing that the ECG-based alignment was not sufficient, and that the sound signal represents a very much lower signal to noise ratio domain.
\end{abstract}

\section{Introduction}

An arrhythmia takes place when regularity is lost or frequency is changed in the heart beats. Those arrhythmias representing risk for the patient require therapy, including anti-arrhythmic drugs, catheter ablation of the arrhythmia mechanisms, cardiac surgery, or implantable devices such as pacemakers or defibrillators [1]. When heart rate is higher than 100 beats-per-minute the arrhythmia is called tachycardia, and when it has ventricular origin, it can produce ventricular fibrillation and sudden cardiac death. During an electrophysiological study (EPS), several catheters are introduced in the patient in order to determine the anatomical location and the functional nature of the arrhythmia mechanism, and then burning under control to suppress the region with abnormal conduction (or lately with extreme cold), so that this ablation ends with the arrhythmia. In some of the cases when tachycardia cancelation is not successful, the patient requires an implantable cardioverter defibrillator (ICD). On the other hand, the physician is supported during the cardiac ablation procedure by several advanced systems, including current cardiac navigation systems (CNS), which allow to replicate the patient cardiac anatomy using a computer program, to guide the catheters precisely within the heart, and to register the cardiac electrical activity with detail [2,-4].

Any previous knowledge about the anatomical location of the ventricular tachycardia origin can contribute to improve the efficacy of the EPS and also to shorten its duration. Previous approaches have mostly used the ECG signals [5], and very recently, the usefulness of the ICD stored electrograms (EGMs) during preceding tachycardia episodes has been scrutinized [6]. However, there are no previous studies analyzing the likely usefulness of the heart sounds to support and contribute to the previous identification of the anatomical location of the ventricular tachycardia origin. Therefore, the present work addressed the signal processing requirements of the cardiac sound signals for their use in this setting.

For this purpose, we designed an advanced signal processing procedure, starting with the usual for ECG or EGM processing, and adapting it to the high-noise nature of the heart-sound signals. In addition, we proposed a spatial- 

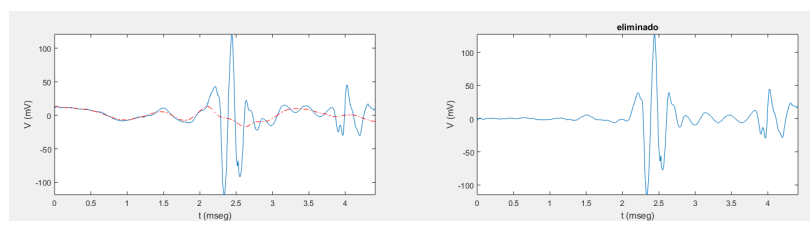

(a)

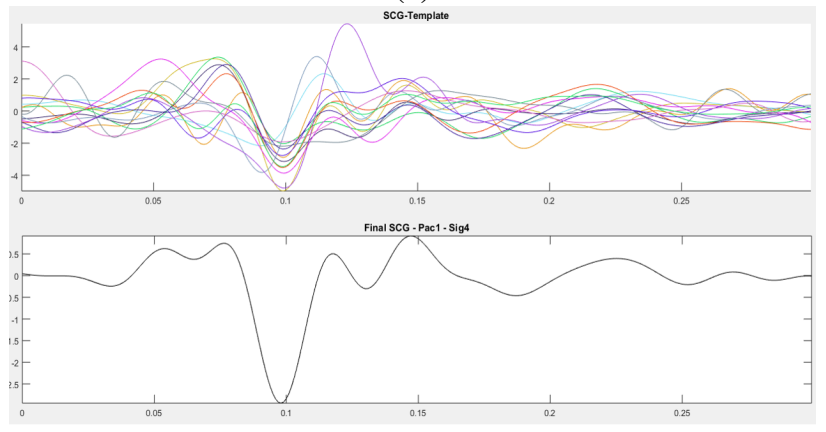

(b)

Figure 1. Signal processing was compared stage by stage on the ECG and on the SCG. (a) Example of suitably adjusted baseline wander removal on the SCG. (b) Overlapped SCG windows after automatic segmentation, and averaged SCG template.

temporal representation for the recorded signals, the socalled M-mode, which allows us to better determine the suitability of the signal processing techniques, by comparison of spatial waveforms in a given location with its neighbors. We finally scrutinized the spatial variability of two features in the heart-sound signals, namely, its amplitude and a newly proposed time-related parameter.

\section{Patients and Database}

The used database was assembled from the patients enrolled in LocMoTIC study [7], which pursuit the analysis of several clinical and technological methods ir order to determine the anatomical origin of ventricular arrhythmia with focal origin. A set of patients with ICD who were also referred to EPS were considered. During the EPS, a protocol was conducted in which pacing trains of 400 ms cycle were delivered from different anatomical locations, the stimulation-catheter coordinates were recorded in the navigation system, and the EGM was recorded in the ICD. EGM signals were recovered after digital processing of printouts, as described in [6].

In addition to the EGM signals, some ICDs are able to record seismocardiogram (SCGs) signals thanks to a microaccelerometer embedded in the tip of a special atrial lead (SonR ${ }^{T M}$ ) whose audible manifestation are the well known heart sounds. The bandwidth provided by this system is larger than conventional phonocardiography, hence, signal components outside the audible band could

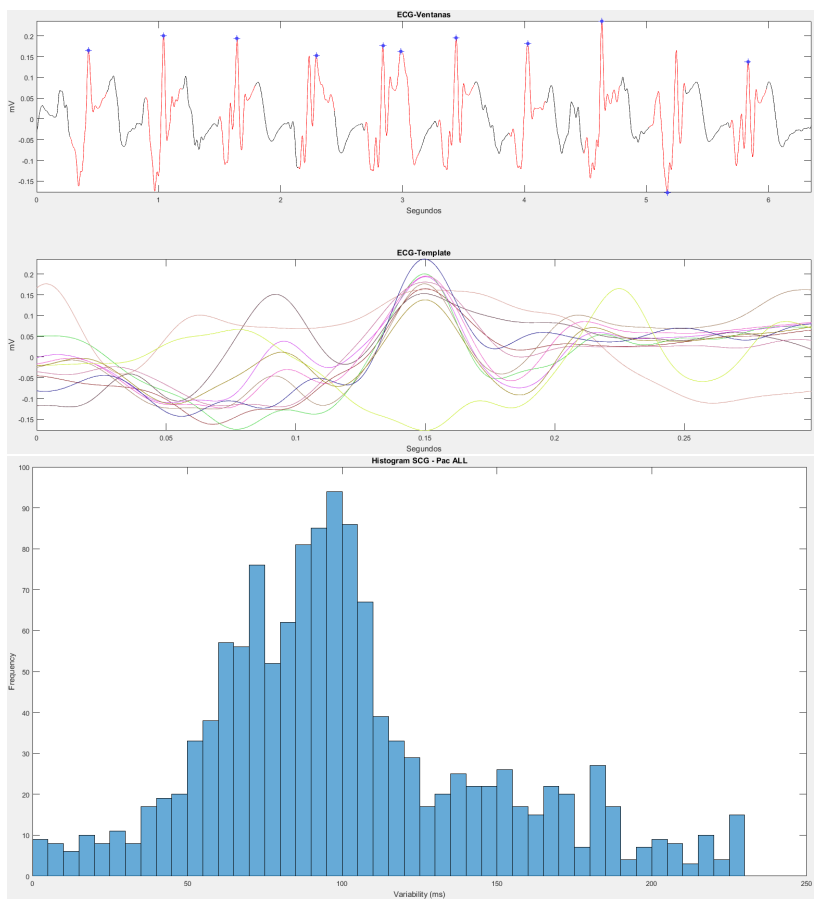

Figure 2. Example of supervised adjustment on the SCG: With the automatic segmentation (up), points in blue are detected as fiducial points, and the misalignment is evident in the segmented waveforms (middle). Histogram (down) of the time-delay for all the patients and all the recordings, after supervised correction.

be recorded [8]. Usually the SonR signal is used to assess the hemodynamic effectiveness of each heart beat as the signal contains significant mechanical and hemodynamic information. However, in the scope of this work, some patients were included in a specific branch of the protocol in order to study the usefulness of the heart rate sounds to support the EGM information. For this purpose, seismocardiogram (SCGs) signals were simultaneously recorded in these patients. When the implanted device was not equipped with SonR ${ }^{T M}$ technology the SCG signals were recorded by means of an accelerometer (high sensitivity $100 \mathrm{mV} / \mathrm{g}$ Bruel\& $\mathrm{Kjaer}^{T M}$ accelerometer for optimized SCG signal acquisition), situated on the medium external region, which was connected to a signal conditioner and amplifier. The SCG signal was recorded in real-time by means of the Biopac $^{T M}$ MP150 system, connected to a dedicated laptop. Specific software allowed to visualize the signals and to export them for subsequent analysis.

In 5 patients, we had available: (1) the basal signals (patient at rest and in sinus rhythm) for the EGM and the SCG; (2) the pacemapping signals, for the EGM and the SCG; (3) at least 3 ECG leads; (4) ventricular meshes, provided by the CNSs, and hence the corresponding coordinates for the pacemapping points. 


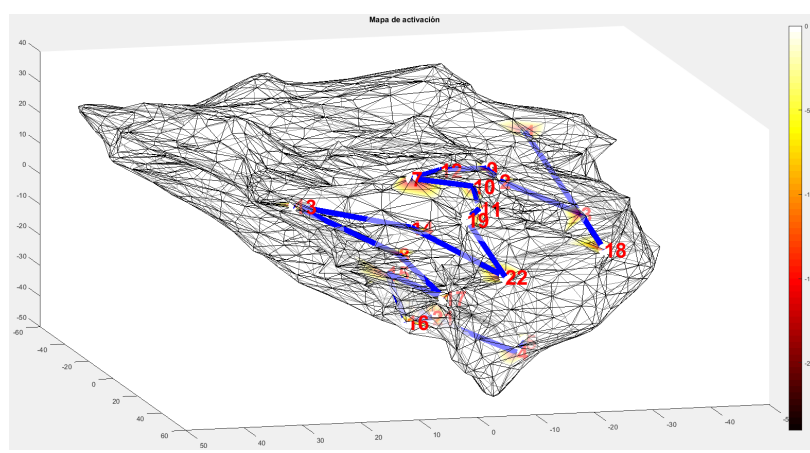

(a)

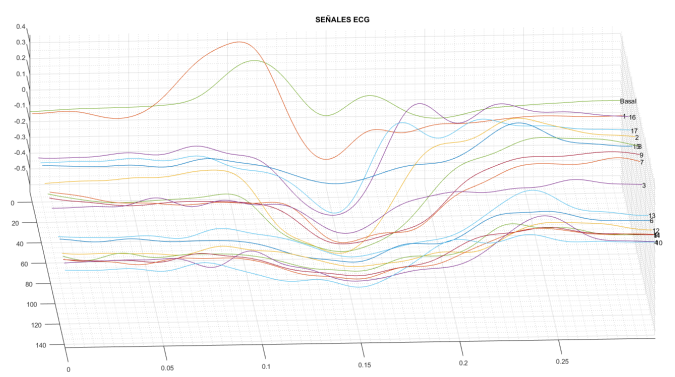

(b)

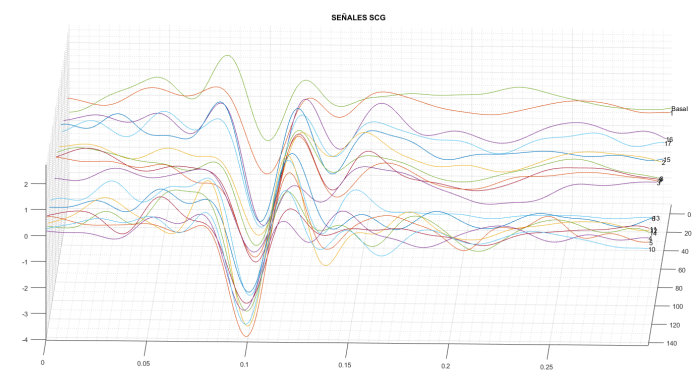

(c)

Figure 3. Spatial-temporal representations for patient 1. (a) Activation map and path for supporting the M-mode. Numbers identify the pacemapping points. (b) M-mode for ECG templates. (c) Idem for SCG templates.

\section{Signal Processing and Results}

Signal processing in ECG and EGM recordings is a well-established research field, and sustained rhythms are often analyzed with temporal filtering, heuristic and morphological processing, and template creation. However, it is not clear that the signal processing for electric signals can be straightforward used in heart sound signals. Therefore, signal processing was compared stage by stage on the ECG and on the SCG signals, taking profit of the fact that both of them had the same time basis at sampling.

Figure 1 shows some relevant observed signals and the processing effect on them. First, signals were low-pass filtered at $40 \mathrm{~Hz}$ for cancelling the high-frequency noise that

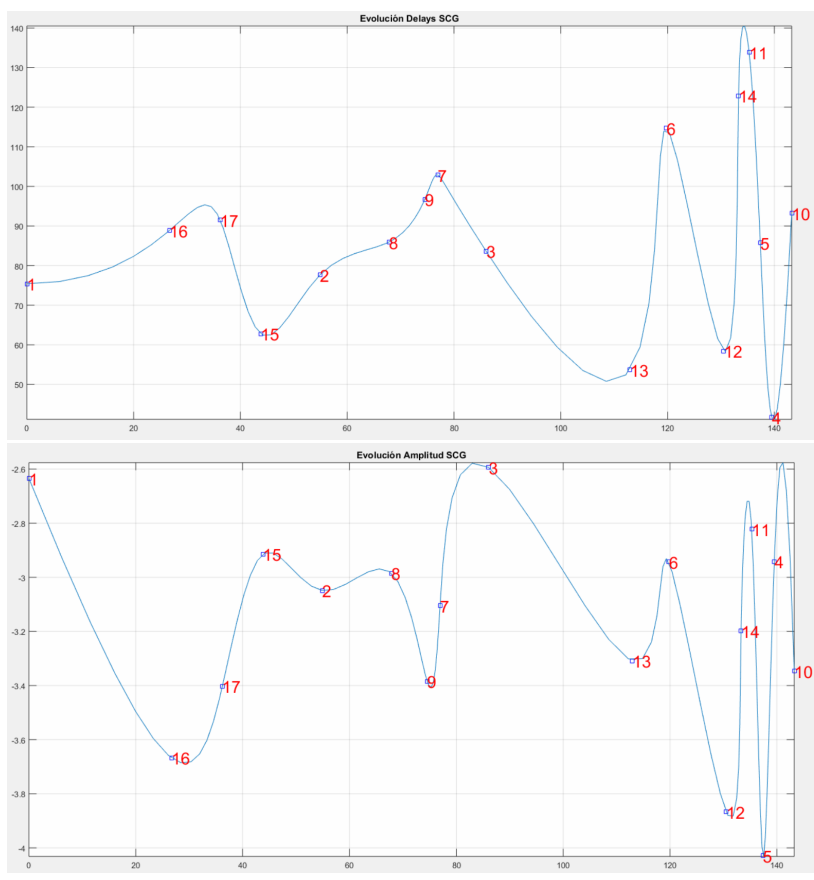

Figure 4. Measured parameters throughout the M-mode spatial line in patient 1: (up) SCG template amplitude; (down) delay of the SCG fiducial point with respect to ECG R-wave.

was present both in the ECG and in the SCG. A notch filter at $50 \mathrm{~Hz}$ and several harmonics was also tested, but it did not give better results for signal conditioning than the low-pass filter. Then, the baseline wander was removed by adjusting a spline to the time-space nodes and obtaining the median of the signal samples at each node. It was observed that a node separation of $700 \mathrm{~ms}$ or higher (up about $1300 \mathrm{~ms}$ ) provided with good results in both signals, whereas lower separation produced strong distortion on the waveforms. The baseline wander was strongly present on the ECG, and smoothly present on the SCG, nevertheless, its suppression improved the SCG signal quality. R waves were detected by using a conventional adaptive threshold approach, but they were obtained only in the ECG signals, and these R-peak times were used both for the ECG and for the SCG in order to segment.

A representative beat was segmented on each $\mathrm{R}$-wave. We used $300 \mathrm{~ms}$ windows centered around the R-peak of the ECG, and the same time window but delayed $150 \mathrm{~ms}$ to include the first sound in the time window. Then, templates were created by averaging beats on each signal. Whereas the ECG templates were consistent during pacemapping, SCG templates were observed to be highly noisy (see Fig. 1c). An automatic algorithm for synchronization was created, which refined the fiducial point of the SCG signal within a moderate exploration window of $\pm 30 \mathrm{~ms}$, which 
did not improve. Nevertheless, the visualization of the fiducial points for each beat in the SCG signal (Fig. $2 \mathrm{a}$ ) showed that two effects were causing the distortion. On the one hand, some of the beats did not exhibit a consistent morphology, which indicated that they were probably non-captured stimulated beats. On the other hand, the phase noise was stronger in SCG than in ECG signals, and hence, a greater beat-to-beat variability is present in sound signals. Therefore, we performed an adjustment on all the SCG signals, by removing the inconsistent beats and by corrected the fiducial point on each beat, according to the situation in neighbor beats for each signals, and on spatially close signals. This improved significantly the quality of the beat templates obtained for the SCG signals.

Histograms were obtained to quantitatively characterize the time jitter in the SCG. For this purpose, the difference was obtained between the synchronization time instants resulting of the R-wave detection in the ECG, with respect to the corrected fiducial point on each SCG beat. Figure $2 \mathrm{p}$ shows the histograms of this correction value calculated on all the signals on the 5 available patients. It can be seen the significant bimodal structure of this parameter, and more, most of its values are in the rank $0-200 \mathrm{~ms}$.

A spatial representation of the left ventricle anatomy was used to plot the nodes, as well as those points in which pacemapping was made, as seen in Fig. 33. These points were ordered along a line with the criteria that all the points were included in the line, and that those points that were closer in the ventricle were also spatially closer along this line (blue). Accordingly, the M-mode was defined as follows: $x$-axis is the Euclidean distance of each point in the blue line with respect to the previous one (in $\mathrm{mm}$ ); $y$-axis is the time (relative to the template); and $z$-axis is the template amplitude, either for the ECG or for the SCG. The name of M-mode is taken from echocardiography, where a exploration line allows to explore the ultrasound velocity in a spatial-temporal domain. The first line is assigned always to the basal (sinus rhythm) template, in order to give a comparison with respect to the ventricular location. Figure 3b shows that a smooth transition can be seen of the ECG waveform changing as a function of space, and that neighbor templates are similar, which only can be observed after the expert alignment. Figure 3 c shows that a remarkable alignment has been also obtained in the SCG templates, even though they are much more noisy templates despite the filtering and averaging. Finally, Fig. 4 shows the spatial evolution of the amplitude and delay parameters for a patient. Note that some of the parameter spatial transitions are smooth and others are notably fast.

\section{Conclusions}

SCG signals exhibit a significantly larger variability than ECG signals. Conventional signal processing for elec- tric signals has to be redesigned if fiducial points are to be estimated. This work has evidenced the relevance of the jitter noise in SCG beats, and the sensitivity of the templates generation to it. Nevertheless, the noise could be partly due to the need for acquiring some external recordings, resulting in increased environmental noise. Hence, the extension of the present work and new works will benefit from the use of intracardiac SCG signals provided by SonR system.

\section{Acknowledgements}

This work was partially supported by Research Grants LocMoTIC, PRINCIPIAS, FINALE, and KERMES (TSI020100-2010-469, TEC2013-48439-C4-1-R, TEC201675161-C2-1-R, and TEC2016-81900-REDT) from Spanish Government. Thanks to Laia Bayarri for valuable help during the recording protocol.

\section{References}

[1] Mann D, Zipes D, Libby P, Bonow R. Braunwald's Heart Disease: A Textbook of Cardiovascular Medicine. 10th edition. Elsevier, 2014.

[2] Izquierdo-Torres MV. Electrophysiological studies with navigation systems. Enferm Cardiol 2005;36:40-44.

[3] Sanromán-Junquera M, Díaz-Valencia R, García-Alberola A, Rojo-Álvarez J, Mora-Jiménez I. Effect of interpolation on electroanatomical mapping. In Computing in Cardiology. 2015; 361-364.

[4] González-Torrecilla E. Navigation systems in current electrophysiology. Rev Esp Cardiol 2004;57:722-724.

[5] Lee S. Classification of ventricular arrhythmia using a support vector machine based on morphological features. In 35 Ann. Int. Conf. IEEE Eng. Med. Biol. Soc. 2013; 57855788.

[6] Sanromán-Junquera M, Mora-Jiménez I, Almendral J, García-Alberola A, Rojo-Álvarez JL. Automatic supporting system for regionalization of ventricular tachycardia exit site in implantable defibrillators. PLoS ONE 2015;10.

[7] Hospitales de Madrid, Sorin Group, and Universidad Rey Juan Carlos. LocMoTiC Project; http://locmotic.org/, Ref TSI-020100-2010-469, 2013.

[8] Tavakolian K, Ngai B, Blaber AP, Kaminska B. Infrasonic cardiac signals: Complementary windows to cardiovascular dynamics. In Proceedings of Engineering in Medicine and Biology Society (IEEE). 2011; 1-4.

Address for correspondence:

José Luis Rojo-Álvarez, D209 (Dep. III) University Rey Juan Carlos. D201, Camino del Molino s/n

28943 - Fuenlabrada (Madrid), Spain

Phone: +34 9148884 64; joseluis.rojo@urjc.es 